SUBJECT AREAS: CONDENSED-MATTER PHYSICS

MATERIALS CHEMISTRY

Received

25 September 2013

Accepted

21 October 2013

Published

6 November 2013

Correspondence and requests for materials should be addressed to

W.P.C. (wpchen@ whu.edu.cn) or Z.J.Y. (ziijieyan@uchicago.

edu)

\title{
Highly mobile and reactive state of hydrogen in metal oxide semiconductors at room temperature
}

\author{
Wan Ping Chen', Ke Feng He', Yu Wang ${ }^{2}$, Helen Lai Wah Chan² \& Zijie Yan ${ }^{3}$
}

\begin{abstract}
'Key Laboratory of Artificial Micro- and Nano-structures of Ministry of Education and School of Physics and Technology, Wuhan University, Wuhan 430072, China, ${ }^{2}$ Department of Applied Physics and Materials Research Centre, The Hong Kong Polytechnic University, Hong Kong, China, ${ }^{3}$ James Franck Institute, The University of Chicago, 929 East 57th Street, Chicago, Illinois 60637 , United States.
\end{abstract}

Hydrogen in metal oxides usually strongly associates with a neighboring oxygen ion through an $\mathrm{O}-\mathrm{H}$ bond and thus displays a high stability. Here we report a novel state of hydrogen with unusually high mobility and reactivity in metal oxides at room temperature. We show that freshly doped hydrogen in $\mathrm{Nb}_{2} \mathrm{O}_{5}$ and $\mathrm{WO}_{3}$ polycrystals via electrochemical hydrogenation can reduce $\mathrm{Cu}^{2+}$ ions into $\mathrm{Cu}^{0}$ if the polycrystals are immersed in $\mathrm{a} \mathrm{CuSO}_{4}$ solution, while this would not happen if the hydrogenated polycrystals have been placed in air for several hours before the immersion. Time-dependent studies of electrochemically hydrogenated rutile single crystals reveal two distinct states of hydrogen: one as protons covalently bonded to oxygen ions, while the other one is highly unstable with a lifetime of just a few hours. Observation of this mobile and reactive state of hydrogen will provide new insight into numerous moderate and low temperature interactions between metal oxides and hydrogen.

$\mathrm{H}$ ydrogen has been attracting extensive research interests aiming at exploring its versatile behaviors in crystalline solids ${ }^{1,2}$. Usually being introduced into metal oxides via annealing in $\mathrm{H}_{2}$ or $\mathrm{H}_{2} \mathrm{O}$-containing atmospheres at elevated temperatures, many interesting, yet sometimes quite complicated features of hydrogen in metal oxides have been revealed ${ }^{3-5}$. They are meaningful for understanding hydrogen incorporated into metal oxides at high temperatures. However, metal oxide semiconductors usually interact with hydrogen at lower or even room temperature in their hydrogen-related applications, such as hydrogenation catalysis ${ }^{6}$, hydrogen storage ${ }^{7,8}$, and low-temperature hydrogen sensing. It is well known that the interaction between metal oxides and hydrogen strongly depends on the reaction temperature. For instance, the reaction of $\mathrm{MO}_{3}(\mathrm{M}=\mathrm{W}$ or $\mathrm{Re})$ with molecular hydrogen at $500^{\circ} \mathrm{C}$ leads to the formation of $\mathrm{MO}_{2}$, while that with dissociated hydrogen at room temperature forms hydrogen bronze $\mathrm{H}_{\mathrm{x}} \mathrm{MO}_{3}{ }^{10}$. It is thus doubtful that the properties of hydrogen obtained from high-temperature hydrogen-doped metal oxides are adequate for understanding low-temperature hydrogenrelated applications of metal oxide semiconductors. As a matter of fact, some investigations have indicated some very puzzling behaviors of hydrogen incorporated into metal oxides at moderate and low temperatures ${ }^{8,11,12}$, and some unusually unstable behaviors have also be observed for hydrogen in those low-temperature hydrogen-related applications of metal oxide semiconductors, yet so far our understanding of these behaviors is very limited ${ }^{13}$.

It is thus highly desirable to study metal oxides with hydrogen introduced at low, and if possible, room temperature. Electrochemical hydrogenation through electrolysis of water has been found to be a facile method to dope hydrogen at room temperature into a number of metal oxides, such as $\mathrm{TiO}_{2}, \mathrm{Nb}_{2} \mathrm{O}_{5}, \mathrm{WO}_{3}, \mathrm{BaTiO}_{3}$ and $\mathrm{Pb}(\mathrm{Zr}, \mathrm{Ti}) \mathrm{O}_{3}{ }^{14-18}$. In this article, we will present some novel findings with regards to the behaviors of hydrogen in metal oxides obtained through this room temperature hydrogen doping method. In "freshly" hydrogenated $\mathrm{Nb}_{2} \mathrm{O}_{5}$ and $\mathrm{WO}_{3}$ polycrystals, a portion of the doped hydrogen apparently exists in a highly mobile and reactive state: a copper layer was deposited on the hydrogenated sample when it was immersed in $\mathrm{CuSO}_{4}$ solution immediately after hydrogenation, while the copper layer would not form if the hydrogenated sample had been aged for ten hours before the immersion. By electrochemically doping hydrogen into $\mathrm{TiO}_{2}(001)$ rutile single crystals, we found a wide step appeared at the low angle side of the (002) peak in X-ray diffraction (XRD); the step quickly narrowed and disappeared in a few hours while the distorted (002) peak (due to hydrogen insertion) would slowly recover over hundreds of hours, and the stretch mode of $\mathrm{O}-\mathrm{H}$ bond was observed by infrared spectroscopy. These contrasts demonstrate two distinct states of hydrogen: one as protons covalently bonded to 
oxygen ions, while the other one being highly mobile and reactive, probably as isolated protons "free" to move in the oxide lattices.

\section{Results}

As illustrated in Fig. 1a, we electrochemically hydrogenated an $\mathrm{Nb}_{2} \mathrm{O}_{5}$ ceramic pellet for $24 \mathrm{~h}$; the white color turned into deep blue due to the incorporation of hydrogen in the oxide lattices ${ }^{15}$. After that, we immediately immersed the pellet in a $0.5 \mathrm{M} \mathrm{CuSO}_{4}$ solution for $10 \mathrm{~min}$ at room temperature. Surprisingly, we observed a reddish layer appeared on the pellet surface, as shown in Fig. 1b. In contrast, if the hydrogenated pellet (another sample) was placed in air for $10 \mathrm{~h}$ at room temperature and then immersed in the $\mathrm{CuSO}_{4}$ solution, no such layer was deposited though it was still deep blue after $10 \mathrm{~h}$ of aging. XRD analysis reveals that the reddish layer is copper as shown in Fig. 1c, and the diffraction peaks of the $\mathrm{Cu}$ are much stronger than those of the $\mathrm{Nb}_{2} \mathrm{O}_{5}$ ceramics. Since $\mathrm{Nb}_{2} \mathrm{O}_{5}$ by itself could not reduce $\mathrm{Cu}^{2+}$ ions as shown in Fig. 1b, the only possible route of the formation of $\mathrm{Cu}$ is the reduction of $\mathrm{Cu}^{2+}$ by hydrogen from the hydrogenated $\mathrm{Nb}_{2} \mathrm{O}_{5}$. What should be emphasized is that in just $10 \mathrm{~min}$ the pellet surface was covered by a thick copper layer, indicating that a relatively large amount of doped hydrogen exists in a highly mobile and reactive state, which could diffuse out of the $\mathrm{Nb}_{2} \mathrm{O}_{5}$ and react with $\mathrm{Cu}^{2+}$ at the surface. The high mobility and reactivity also imply a short lifetime of such hydrogen, and after $10 \mathrm{~h}$ of aging they almost disappeared from the oxide lattices so no copper was deposited on the aged pellet during the immersion.

$\mathrm{Nb}_{2} \mathrm{O}_{5}$ is not the only oxide that can accommodate such highly mobile and reactive hydrogen. Indeed copper was also found to be deposited on a 24 h electrochemically hydrogenated $\mathrm{WO}_{3}$ pellet when it was immersed in $0.5 \mathrm{M} \mathrm{CuSO}_{4}$ solution for $10 \mathrm{~min}$ immediately after the hydrogenation, but the amount was much less than that formed on similarly hydrogenated $\mathrm{Nb}_{2} \mathrm{O}_{5}$ (see the XRD pattern in Fig. 1c). As a large amount of hydrogen can be electrochemically inserted into $\mathrm{WO}_{3}$, it is strange that only a little portion of inserted hydrogen seems to exist in the mobile and reactive state so the amount of deposited copper was very small. These results suggest that this special state of hydrogen may be not rare among metal oxides, while its concentration can be dramatically different among different metal oxides.
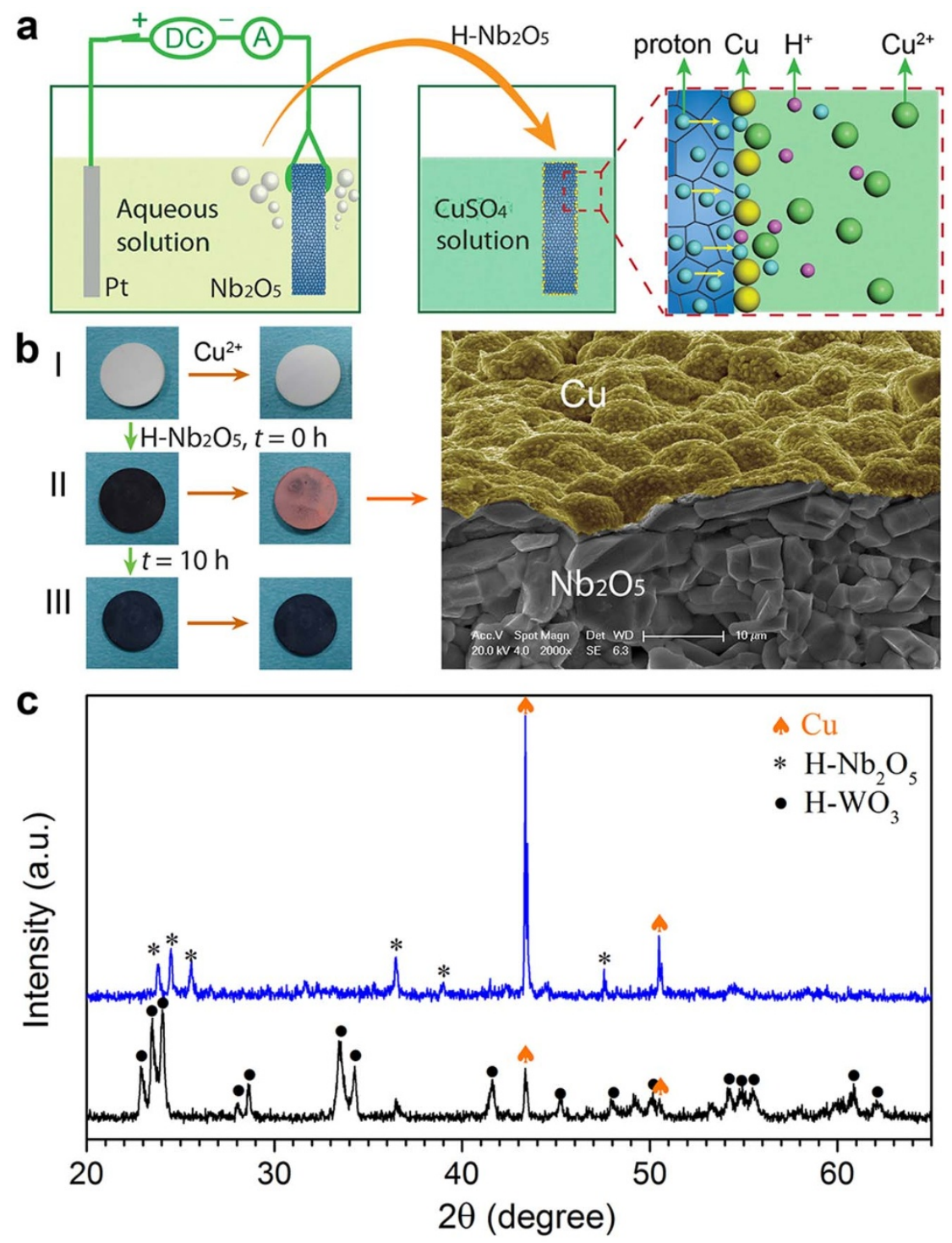

Figure 1 Highly reactive hydrogen in hydrogenated oxides. (a), Schematic of electrochemically hydrogenating an $\mathrm{Nb}_{2} \mathrm{O}_{5}$ ceramic pellet and then immersing it into a $0.5 \mathrm{M} \mathrm{CuSO}_{4}$ solution at room temperature. (b), Optical images of $\mathrm{Nb}_{2} \mathrm{O}_{5}$ pellets before and after being immersed in $0.5 \mathrm{M} \mathrm{CuSO}_{4}$ solution for $10 \mathrm{~min}$ : I. an as-sintered sample, II. a hydrogenated sample, III. a hydrogenated and $10 \mathrm{~h}$-aged sample. The right side is a scanning electron microscopy image taken over the cross-section of an immersed sample. The Cu layer is shown with a false color. c, XRD pattern taken on the surface of the hydrogenated $\mathrm{Nb}_{2} \mathrm{O}_{5}$ pellet after 10 min immersion. A pattern taken on the surface of a hydrogenated $\mathrm{WO}_{3}$ pellet after 10 min immersion is also shown. 
We expect that it should be possible to reveal a more detailed evolution of this unstable hydrogen in metal oxide single crystals. As $\mathrm{Nb}_{2} \mathrm{O}_{5}$ and $\mathrm{WO}_{3}$ single crystals are not available, we turned to $\mathrm{TiO}_{2}$ rutile single crystals to conduct time-dependent study. These single crystals have nearly perfect crystal lattices that can eliminate the influence of crystal defects to doped hydrogen. For some metal oxides, phase transformation or crystal reformation occurs upon the hydrogen doping ${ }^{16}$. In this study, although we have tried to insert as much hydrogen as possible into the rutile single crystals, no new phases could be detected through XRD. Instead, the lattice of the rutile single crystals was found to be distorted to an extent beyond expectation when much hydrogen was inserted. Figure 2a shows XRD patterns of a hydrogenated rutile single crystal measured at different aging times, $t$, after $200 \mathrm{~h}$ of electrochemical hydrogen insertion. The (002) peak of as-received single crystals is sharp and

a

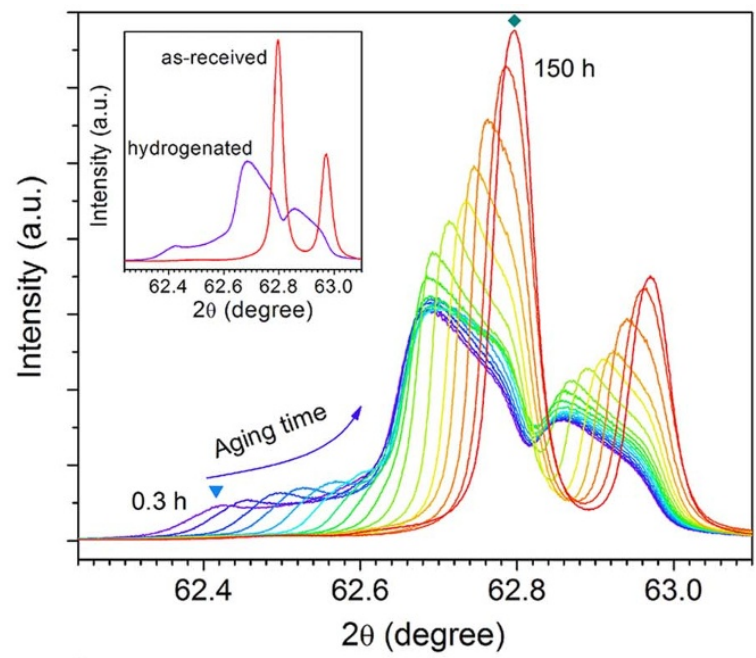

b

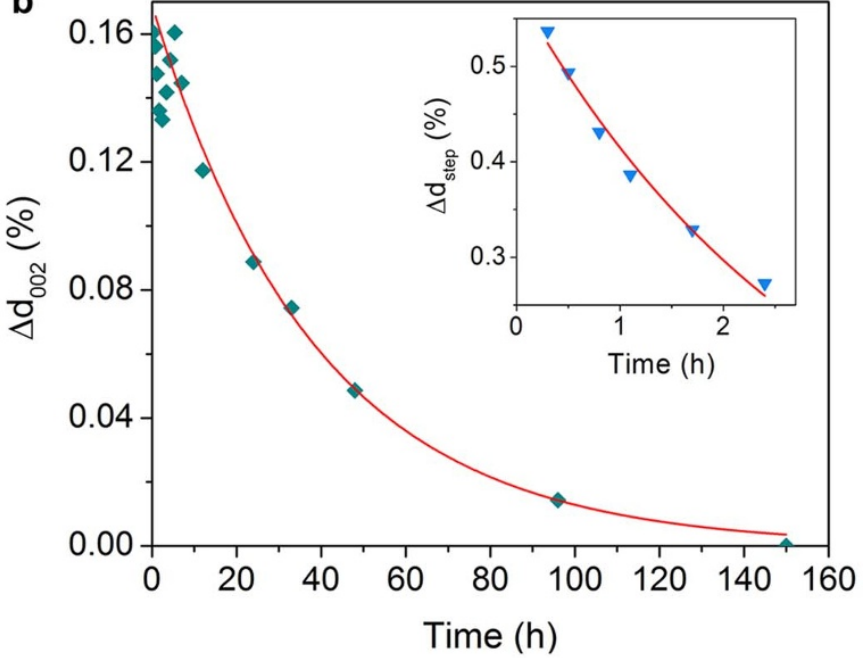

Figure $2 \mid$ Crystal structure evolution of electrochemically hydrogenated $\mathrm{TiO}_{2}$. (a), XRD patterns of an electrochemically hydrogenated (001) rutile single crystal taken after a series of aging time. The hydrogenation time is $200 \mathrm{~h}$, and the aging times are $0.3,0.5,0.8,1.1,1.7,2.4,3.4,4.3,5.4,7,12$, $24,33,48,96$, and $150 \mathrm{~h}$ for the patterns from left to right. Inset: XRD patterns of an as-received (001) rutile single crystal and the electrochemically hydrogenated (001) rutile single crystal measured at an aging time of $0.3 \mathrm{~h}$. (b), Lattice expansion of the (002) plane of the hydrogenated crystal compared to that of the undoped one as a function of aging time. The red curve is an exponential fit of the data: $\Delta d=$ $0.0017 \exp (-t / 39)$. Inset: the lattice expansion of the wide step indicated by a triangle in panel a. The red curve is also an exponential fit: $\Delta d=$ $0.0058 \exp (-t / 3)$ highly symmetric (see the inset). In contrast, the (002) diffraction peak of the hydrogenated crystal becomes broad and is distorted to a very irregular shape, indicating non-uniform plane spacing or hydrogen distribution. An important observation is that there exists a wide step at the low angle side of the peak for short aging times, e.g., at $62.42^{\circ}$ as indicated by the triangle for $t=0.3 \mathrm{~h}$. We note that while the specific shape of the (002) peak could vary depending on the hydrogen insertion time, the broadening of the peak and the arising of a wide step always occur (see Supplementary Information, Fig. S1). Chester and Bradhurst have electrochemically inserted hydrogen into vacuum-reduced rutile single crystals and found that the hydrogen concentration gradient was much higher in the first few microns of the surface layer than in the bulk region $(\sim 100 \mu \mathrm{m} \text { deep })^{19}$. The wide step revealed in our study can be well explained in term of this layered structure: the concentration of hydrogen is particularly high at the surface, where the plane spacing is expanded by $0.54 \%$ at $t=$ $0.3 \mathrm{~h}$ compared to that of the undoped (002) plane, while at the same time the expansion of the inner layer in the bulk region is only $0.16 \%$ as indicated by the distorted peak.

The XRD patterns further reveal that the distortion evolved with the aging time by placing the hydrogenated crystals in air at room temperature, indicating that the distorted crystal lattice as well as the layered structure subsequently experiences a spontaneous and systematic restoration as shown in Fig. 2b. The lattice expansion, $\Delta d$, of the hydrogenated crystal decreases exponentially by

$$
\Delta d=\Delta d_{0} \exp (-t / \tau)
$$

where $\Delta d_{0}$ is the initial lattice expansion and $\tau$ is a decay constant. For the case of the wide step, $\Delta d_{0}=0.58 \%$ and $\tau=3 \mathrm{~h}$, indicating that the layered structure exists only for a few hours in our sample after the hydrogen insertion, and thus the hydrogen in the layer is highly mobile. In contrast, it takes longer aging time for the distorted peak to restore to the original state. The distorted (002) peak in Fig. 2a gradually decreases in width, and increases in height and symmetry following the eq. 1 with $\Delta d_{0}=0.17 \%$ and $\tau=39 \mathrm{~h}$. After an aging time of $t=150 \mathrm{~h}$, the peak has almost restored to its original highly sharp and symmetric shape. Spontaneous recovery was also observed for other initial distortions (Supplementary Information, Fig. S2). These two dramatically different decay constants exactly reflect the obvious difference in mobility observed for the step and the peak. The difference suggests that hydrogen in the surface layer is not only of a higher concentration but also much more mobile than that in the bulk region, so there exist two distinct states of hydrogen with different mobilities in these electrochemically hydrogenated rutile single crystals. This is consistent with the observations that only the "freshly" hydrogenated $\mathrm{Nb}_{2} \mathrm{O}_{5}$ and $\mathrm{WO}_{3}$ polycrystals could reduce $\mathrm{Cu}^{2+}$ ions but those aged samples could not. In other words, highly unstable hydrogen has been observed in all these three metal oxides that were investigated in this study, although its concentration is dramatically different in different metal oxides.

Figure 3 shows infrared absorption spectra measured at $300 \mathrm{~K}$ for a rutile single crystal that has been electrochemically hydrogenated for $120 \mathrm{~h}$ and an as-received rutile single crystal. The as-received rutile single crystals are colorless and almost transparent without clear absorption peak in the range of $3100-3400 \mathrm{~cm}^{-1}$ that is common for as-grown rutile single crystals. In contrast, the hydrogenated sample was deep-blue in color and its transparency was greatly decreased. A strong and broad absorption peak centered at $3279 \mathrm{~cm}^{-1}$ can be clearly observed. This peak is continuously weakened with increasing aging time and meanwhile the transmittance of the hydrogen-inserted rutile single crystal is increased. It is well known that the infrared absorption peak at $3279 \mathrm{~cm}^{-1}$ is due to the stretch mode of $\mathrm{O}-\mathrm{H}$ bond at room temperature in rutile. So this peak can be assigned to the stretch mode of $\mathrm{O}-\mathrm{H}$ bond ${ }^{20}$. As a matter of fact, electrochemically doped hydrogen has been found to greatly 


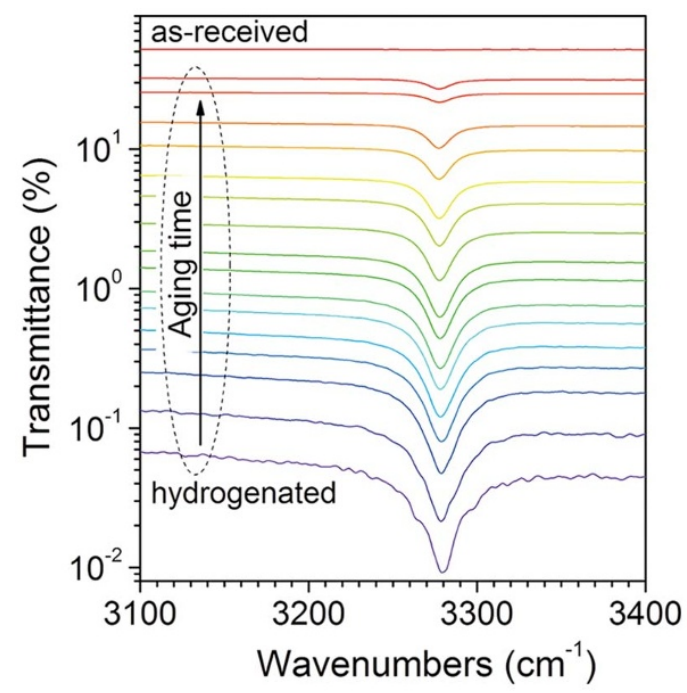

Figure $3 \mid$ Infrared absorption spectra of an as-received rutile single crystal and a hydrogenated one measured after a series of aging times. The hydrogenation time is $120 \mathrm{~h}$, and the aging times are 2, 9, 24, 34, 48, 72, $144,192,310,500,750,1150,1550,2400$, and $3000 \mathrm{~h}$ for the spectra from bottom to top.

increase the conductivity and act as a shallow donor in rutile single crystals $^{21}$. No other hydrogen-related structures can be observed over a wide frequency range of $500-4000 \mathrm{~cm}^{-1}$ (Supplementary Information, Fig. S3), and it is necessary to point out that hydrogen can exist in metal oxides in some forms that are not seen by IR spectroscopy ${ }^{22,23}$. Obviously, for the two distinct states of hydrogen revealed by XRD, the one with a bigger decay constant can be identified as protons covalently bound to oxygen ions according to the infrared absorption spectra analysis, which can be expressed as $(\mathrm{OH})_{\mathrm{O}}^{\bullet 24}$; while the highly mobile one is still largely unknown, e.g., its charge state that can be either positive, neutral or negative ${ }^{25}$. For simplicity, we tentatively assume a positive charge state for it and term it as $H_{i m}^{\bullet}$ ( $i$ for interstitial and $m$ for mobile) hereinafter.

\section{Discussion}

Hydrogen existing as $(\mathrm{OH})_{O}^{\bullet}$ is well-known for metal oxides and is found in many applications of metal oxides. For example, for electrochromic metal oxide $\mathrm{WO}_{3},(\mathrm{OH})_{\mathrm{O}}^{\bullet}$ is charged/discharged reversibly through controlling applied voltage ${ }^{10}$, which can be expressed using the notation of Kröger and Vink in the following way:

$$
H^{+}(a)+e^{\prime}+W_{W}^{\times}+O_{O}^{\times} \Leftrightarrow W_{W}^{\prime}+(O H)_{O}^{\bullet},
$$

where $W_{W}^{\prime}$ represents a $\mathrm{W}^{5+}$ at the lattice site of $\mathrm{W}^{6+}$ in $\mathrm{WO}_{3}$ and $H^{+}(a)$ a proton in a solution. The electrochromic effect is directly related the formation of $W_{W}^{\prime}$, namely $\mathrm{W}^{5+}$ in the lattice. It should be pointed out that the formation of $\mathrm{O}-\mathrm{H}$ bond tends to weaken the ferroelectric distortion in $\mathrm{WO}_{3}{ }^{10}$ and with increasing content of $(\mathrm{OH})_{\mathrm{O}}^{\bullet}$, the crystal structure is modified from monoclinic $\left(\mathrm{WO}_{3}\right)$ to tetragonal $\left(\mathrm{H}_{0.1} \mathrm{WO}_{3}\right.$ and $\left.\mathrm{H}_{0.33} \mathrm{WO}_{3}\right)$, and to cubic $\left(\mathrm{H}_{0.5} \mathrm{WO}_{3}\right)^{16}$. Through thermal treatment, it leaves from the lattice of $\mathrm{WO}_{3}$ as water ${ }^{26}$ :

$$
2(\mathrm{OH})_{\mathrm{O}}^{\bullet} \rightarrow \mathrm{O}_{\mathrm{O}}^{\times}+\mathrm{V}_{\mathrm{O}}^{\bullet \bullet}+\mathrm{H}_{2} \mathrm{O} .
$$

In contrast, as a different state, hydrogen existing as $H_{i m}^{\bullet}$ is expected to leave the oxide lattice in a different form rather than $\mathrm{H}_{2} \mathrm{O}$. A mass spectroscopy analysis of the gas released from electrochemically hydrogenated $\mathrm{Nb}_{2} \mathrm{O}_{5}$ upon thermal treatment included a large portion of $\mathrm{H}_{2}$ besides $\mathrm{H}_{2} \mathrm{O}^{8,26}$. Considering that $\mathrm{H}_{2} \mathrm{O}$ is formed from
$(\mathrm{OH})_{\mathrm{O}}^{\bullet}, \mathrm{H}_{2}$ should be formed from $H_{i m}^{\bullet}$, which exists in electrochemically hydrogenated $\mathrm{Nb}_{2} \mathrm{O}_{5}$, upon thermal treatment in the following way:

$$
2 H_{i m}^{\bullet}+2 e^{\prime} \rightarrow H_{2}(g),
$$

where the electron is weakly-bonded or free in the metal oxide and the out-diffusion of hydrogen decreases the concentration of charge carriers and thus the conductivity of metal oxides. As for the deposition of copper on "freshly" hydrogenated $\mathrm{Nb}_{2} \mathrm{O}_{5}$ and $\mathrm{WO}_{3}$ pellets when being immersed in the $\mathrm{CuSO}_{4}$ solution, it reveals the following reaction between $H_{i m}^{\bullet}$ and $\mathrm{Cu}^{2+}$ at room temperature:

$$
2 H_{i m}^{\bullet}+2 e^{\prime}+\mathrm{Cu}^{2+}(a) \rightarrow C u(s)+2 H^{+}(a) .
$$

As $H_{i m}^{\bullet}$ can be of a high concentration in hydrogenated $\mathrm{Nb}_{2} \mathrm{O}_{5}$, a large portion of $\mathrm{H}_{2}$ is formed besides $\mathrm{H}_{2} \mathrm{O}$ upon thermal treatment, and a large amount of copper is formed when immersed in $\mathrm{CuSO}_{4}$ solution at room temperature. On the other hand, since the content of $H_{i m}^{\bullet}$ is very small in electrochemically hydrogenated $\mathrm{WO}_{3}$, it is difficult to collect $\mathrm{H}_{2}$ upon heat-treatment and only $\mathrm{H}_{2} \mathrm{O}$ can be detected, and the amount of copper deposition is very small when immersed in $\mathrm{CuSO}_{4}$ solution.

It is clear that with such surprisingly high mobility and reactivity at room temperature, this special state of hydrogen will not be observed or studied in samples treated through hydrogen-doping at elevated temperatures. Our finding of the highly mobile and reactive state of hydrogen, and this room-temperature hydrogen doping method as well, are thus especially valuable for studying those interactions between metal oxides and hydrogen at moderate and low temperatures. For example, the peculiar aging behavior of electrochemically hydrogenated $\mathrm{TiO}_{2}$ rutile single crystals ${ }^{14,21}$, and some other metal oxides as well ${ }^{12,18,27}$, can now be better understood: the mobile hydrogen $H_{i m}^{\bullet}$ should play an important role in the initial stage of aging, while $(\mathrm{OH})_{\mathrm{O}}^{\bullet}$ should be mainly responsible for the subsequent aging $^{28}$. For hydrogen-sensing of metal oxide semiconductors, it is generally considered that the change of electrical resistance with hydrogen exposure is only related to the spilt hydrogen atoms on the surface $e^{9,29,30}$, in this way electron transfer from hydrogen to metal oxides occurs while hydrogen is still highly mobile. According to our present results, however, ionized hydrogen, or proton, can actually exist inside metal oxides while being highly mobile. So it is necessary to examine whether hydrogen will enter into the lattice of metal oxides in the course of hydrogen-sensing. Similarly, hydrogen entrance into the lattice of metal oxides should also be considered for other chemisorption of atomic hydrogen on the surface of metal oxides ${ }^{31,32} . \mathrm{Nb}_{2} \mathrm{O}_{5}$ is so far the most effective oxide promoter for accelerating adsorption/desorption of hydrogen in magnesium hydride storage materials. It has been assumed that $\mathrm{Nb}_{2} \mathrm{O}_{5}$ may play the role as a reservoir loading a certain amount of split hydrogen in its lattice in the course of catalysis ${ }^{8,11,26}$. Obviously, our results provide a direct support for this assumption and more attention should be paid to it when the catalysis mechanism of $\mathrm{Nb}_{2} \mathrm{O}_{5}$ is studied.

In summary, by studying electrochemically hydrogenated $\mathrm{Nb}_{2} \mathrm{O}_{5}$ and $\mathrm{WO}_{3}$ polycrystals, and $\mathrm{TiO}_{2}$ rutile (001) single crystals, we found the coexistence of hydrogen in two distinct states inside the lattice of these metal oxides: one is as protons attached to oxygen ions by strong $\mathrm{O}-\mathrm{H}$ bonds while the other one is highly mobile and reactive, but exists only for several hours in the lattice. The properties of this mobile and reactive hydrogen are still largely unknown, and further investigations need to be conducted on this special state of hydrogen, such as what its actual charge state is, how $H_{i m}^{\bullet}$ interacts with metal oxide lattices, how $H_{i m}^{\bullet}$ interacts with other crystal defects like $V_{O}^{\bullet \bullet}$, and how, if possible, $H_{i m}^{\bullet}$ and $(\mathrm{OH})_{O}^{\bullet}$ are transformed into each other. Our results are therefore important for deepening our understanding of hydrogen in metal oxides and for promoting numerous hydrogenrelated applications of metal oxides. 


\section{Methods}

$\mathrm{Nb}_{2} \mathrm{O}_{5}$ samples were prepared through $2 \mathrm{~h}$ sintering at $1250^{\circ} \mathrm{C}$ in air of pellets ( $15 \mathrm{~mm}$ in diameter and $2 \mathrm{~mm}$ thick) pressed from analytical grade $(99 \%) \mathrm{Nb}_{2} \mathrm{O}_{5}$ powders. Analytical grade $(99 \%) \mathrm{WO}_{3}$ powder was first ball milled with de-ionized water for $24 \mathrm{~h}$. After drying, the milled powder was pressed into pellets of $15 \mathrm{~mm}$ in diameter and $2 \mathrm{~mm}$ thick and the pellets were sintered at $1150^{\circ} \mathrm{C}$ for $2 \mathrm{~h}$ in air. The $\mathrm{TiO}_{2}$ rutile single crystals used in this work were (001)-oriented $10 \times 10 \times 0.5 \mathrm{~mm}^{3}$ commercial wafers purchased from HEFEI KEJING (China). Two $2 \mathrm{~mm}$ diameter silver electrodes were fired on the two sides of a corner of the single crystals. Electrochemical hydrogen insertion was conducted by immersing a sample in a $0.01 \mathrm{M} \mathrm{NaOH}$ solution. The $\mathrm{Nb}_{2} \mathrm{O}_{5}$ or the $\mathrm{WO}_{3}$ pellets or the silver electrodes of the rutile single crystals were connected to dc voltages $(6 \mathrm{~V})$ to act as the cathode to electrolyze water. Infrared absorption spectra were recorded with a Nicolet iS-10 Fourier transform spectrometer by measuring the transmittance of as-received and hydrogenated rutile single crystals at $300 \mathrm{~K}$. The spectral resolution was $0.5 \mathrm{~cm}^{-1}$. $\mathrm{X}$-ray diffraction patterns were taken through an X-ray diffractometer (Philips PW 3719) with $\mathrm{Cu} \mathrm{K} \alpha$ radiation.

1. Van de Walle, C. G. Hydrogen as a cause of doping in zinc oxide. Phys Rev Lett $\mathbf{8 5}$ 1012-1015 (2000).

2. Kilic, C. \& Zunger, A. n-type doping of oxides by hydrogen. Appl Phys Lett 81, 73-75 (2002).

3. Shi, G. A. et al. Hydrogen local modes and shallow donors in ZnO. Phys Rev B 72, 195211 (2005)

4. Spahr, E. J. et al. Giant of hydrogen transport in rutile $\mathrm{TiO}_{2}$ at low temperatures. Phys Rev Lett 104, 205901 (2010).

5. Tarun, M. C. \& McCluskey, M. D. Infrared absorption of hydrogen-related defects in strontium titanate. J Appl Phys 109, 063706 (2011).

6. Marcq, J. P., Poncelet, G. \& Fripiat, J. J. Hydrogenation by hydrogen bronzes: II Hydrogenation of ethylene by $\mathrm{H}_{\mathrm{x}} \mathrm{V}_{2} \mathrm{O}_{5}$. J Catal 87, 339-351 (1984).

7. Berzins, A. R. \& Sermon, P. A. Reversible isothermal sorption of hydrogen by tungsten trioxide in presence of platinum. Nature 303, 506-508 (1983).

8. Dolci, F., Di Chio, M., Baricco, M. \& Giamello, E. Niobium pentoxide as promoter in the mixed $\mathrm{MgH}_{2} / \mathrm{Nb}_{2} \mathrm{O}_{5}$ system for hydrogen storage: a multitechnique investigation of the $\mathrm{H}_{2}$ uptake. J Mater Sci 42, 7180-7185 (2007).

9. Varghese, O. K. et al. Extreme changes in the electrical resistance of titania nanotubes with hydrogen exposure. Adv Mater 15, 624-627 (2003).

10. Dickens, P. G., Crouchbaker, S. \& Weller, M. T. Hydrogen insertion in oxides. Solid State Ionics 18-9, 89-97 (1986).

11. Borgschulte, A., Rector, J. H., Dam, B., Griessen, R. \& Zuttel, A. The role of niobium oxide as a surface catalyst for hydrogen absorption. J Catal 235, 353-358 (2005).

12. Yao, W. Q. et al. Hydrogen-induced degradation and aging of $\mathrm{Pb}\left(\mathrm{Mg}_{1 / 3} \mathrm{Nb}_{2 / 3}\right) \mathrm{O}_{3^{-}}$ based X7R multilayer ceramic capacitors. Jpn J Appl Phys 47, 5530 (2008).

13. Kreuer, K.-D. Proton conductivity: materials and applications. Chem Mater 8 , 610-641 (1996).

14. Chen, W. P. et al. Spontaneous recovery of hydrogen-degraded $\mathrm{TiO}_{2}$ ceramic capacitors. Appl Phys Lett 84, 103-105 (2004).

15. Chen, W. P. et al. Hydrogen as an unstable shallow donor in oxides. Jpn J Appl Phy 49, 051103 (2010)

16. Chen, W. P. et al. Effects of electrochemical hydrogen charging on electrical properties of $\mathrm{WO}_{3}$ ceramics. J Mater Sci 42, 2524-2527 (2007).

17. Chen, W. P., Jiang, X. P., Wang, Y., Peng, Z. \& Chan, H. L. W. Water-induced degradation of barium titanate ceramics studied by electrochemical hydrogen charging. J Am Ceram Soc 86, 735-737 (2003).

18. Shafiei, A., Nickchi, T., Oprea, C., Alfantazi, A. \& Troczynski, T. Investigation of hydrogen effects on the properties of $\mathrm{Pb}(\mathrm{Zr}, \mathrm{Ti}) \mathrm{O}_{3}$ in tetragonal phase using water electrolysis technique. Appl Phys Lett 99, 212903 (2011).

19. Chester, P. F. \& Bradhurst, D. H. Electrolytically induced conductivity in rutile. Nature 199, 1056 (1963).
20. Johnson, O. W., Paek, S. H. \& DeFord, J. W. Diffusion of $\mathrm{H}$ and $\mathrm{D}$ in $\mathrm{TiO}_{2}$ suppression of internal fields by isotope exchange. J Appl Phys 46, 1026 (1975).

21. Chen, W. P., Wang, Y. \& Chan, H. L. W. Hydrogen: a metastable donor in $\mathrm{TiO}_{2}$ single crystals. Appl Phys Lett 92, 112907 (2008).

22. Fillaux, F., Ouboumour, H., Tomkinson, J. \& Yu, L. T. An inelastic neutron scattering study of the proton dynamics in $\gamma-\mathrm{MnO}_{2}$. Chem Phys 149, 459-469 (1991).

23. Shi, G. A., Saboktakin, M., Stavola, M. \& Pearton, S. J. "Hidden hydrogen" in asgrown ZnO. Appl Phys Lett 85, 5601-5603 (2004).

24. Iwahara, H. in: Colomban, P. (ed).Proton Conductors: Solids, Membranes, and Gels: Materials and Devices. Cambridge University Press, New York, 1992.

25. Kobayashi, Y. et al. An oxyhydride of $\mathrm{BaTiO}_{3}$ exhibiting hydride exchange and electronic conductivity. Nature Mater 11, 507-511 (2012).

26. Dolci, F., Di Chio, M., Baricco, M. \& Giamello, E. The interaction of hydrogen with oxidic promoters of hydrogen storage in magnesium hydride. Mater Res Bull 44, 194-197 (2009)

27. Chen, W. P., Wang, Y., Chan, H. L. W. \& Luo, H. S. Hydrogen-related dynamic dielectric behavior of barium titanate single crystals. Appl Phys Lett 88, 202906 (2006).

28. Herklotz, F., Lavrov, E. V. \& Weber, J. Infrared absorption of the hydrogen donor in rutile $\mathrm{TiO}_{2}$. Phys Rev B 83, 235202 (2011).

29. Lu, C., Chen, Z. \& Yu, P. A novel reaction model for the electrical conductivity of ultra-thin $\mathrm{TiO}_{2}$ films in $\mathrm{H}_{2}$. Phys Chem Chem Phys 13, 9131-9133 (2011).

30. Wang, Z. et al. Fast and highly-sensitive hydrogen sensing of $\mathrm{Nb}_{2} \mathrm{O}_{5}$ nanowires at room temperature. Intl J Hydrogen Energy 37, 4526-4532 (2012).

31. Roland, U., Braunschweig, T. \& Roessner, F. On the nature of spilt-over hydrogen. J Mol Catal A: Chem 127, 61-84 (1997).

32. Roland, U., Salzer, R., Braunschweig, T., Roessner, F. \& Winkler, H. Investigations on hydrogen spillover: Part 1.-electrical conductivity studies on titanium dioxide. J Chem Soc-Faraday Trans 91, 1091-1095 (1995).

\section{Acknowledgments}

The authors thank Prof. Yuan Zhi Li for his valuable discussion. This work was supported by the National High Technology Research and Development Program of China (863 Program) (No.2013AA031903), the National Basic Research Program of China under Grant No. 2009CB939705, and the National Natural Science Foundation of China under Grant No. J1210061 and 50772077.

\section{Author contributions}

W.P.C. designed the research and conducted the experiments. W.P.C. and Z.Y. wrote the manuscript. K.F.H. contributed to the measurements. Z.Y., Y.W. and H.L.W.C. contributed to the project design and data analysis. All authors discussed the results and commented on the paper.

\section{Additional information}

Supplementary information accompanies this paper at http://www.nature.com/ scientificreports

Competing financial interests: The authors declare no competing financial interests.

How to cite this article: Chen, W.P., He, K.F., Wang, Y., Chan, H.L.W. \& Yan, Z.J. Highly mobile and reactive state of hydrogen in metal oxide semiconductors at room temperature. Sci. Rep. 3, 3149; DOI:10.1038/srep03149 (2013).

This work is licensed under a Creative Commons Attribution 3.0 Unported license. To view a copy of this license, visit http://creativecommons.org/licenses/by/3.0 